\title{
Test de Fluidez Verbal: datos normativos y desarrollo evolutivo en el alumnado de primaria
}

\author{
Eduardo García, Cristina Rodríguez, Raquel Martín, Juan E. Jiménez, \\ Sergio Hernández y Alicia Díaz \\ Universidad de La Laguna (España)
}

\begin{abstract}
El objetivo principal de este estudio consistió en la baremación y el análisis del desarrollo evolutivo, en una amplia muestra de población infantil de habla española, de dos pruebas destinada a la evaluación de la fluidez verbal (semántica y fonológica). Analizamos el desarrollo de ambas tareas dentro de un contexto de diseño transversal. Se seleccionó una muestra de 1.032 sujetos con edades comprendidas entre los 6 años y 3 meses y los 12 años y cuatro meses que cursaban entre primero y sexto de educación primaria. La fluidez verbal semántica se evaluó con la tarea de evocación 'de animales en un minuto' y la fluidez verbal fonológica se evaluó con las consignas ' $\mathrm{F}, \mathrm{A}, \mathrm{M}$ '. Los resultados muestran una mejora progresiva en el rendimiento en función de la edad en ambas pruebas. Se presentan tablas de medias, desviación estándar y percentiles para los distintos grupos de edad.
\end{abstract}

Palabras clave: Baremación, desarrollo cognitivo, funciones ejecutivas, fluidez verbal semántica, fluidez verbal fonológica.

Verbal Fluency Test: normative data and evolutionary development in elementary students. The aim of this study was the standardization, and analysis of the developmental profile of two tasks for the assessment of verbal fluency (semantic and phonological), in a large sample of Spanish-speaking child population. We analyzed the development of both tasks within a context of cross-sectional design. A selected a sample of 1.032 subjects aged between 6 years and 3 months to 12 years and four months between first and sixth grade were of primary education. The semantic verbal fluency was evaluated with the task 'animals in 1 minute' and the phonologic verbal fluency was evaluated with 'F, A, M' in a minute for each one'. The results show a progressive improvement in performance as a function of age in both tests. We present tables of means, standard deviations and percentiles for different age groups.

Key words: Standardization, cognitive development, executive functions, semantic verbal fluency, phonological verbal fluency.

Correspondencia: Eduardo García. Departamento de Psicología Evolutiva y de la Educación. Universidad de La Laguna. Campus de Guajara, C.P. 38205. La Laguna, Tenerife (España). Email: egarciam@ull.es 
Cuando preguntamos a alguien que diga todas las palabras que pueda a partir de una consigna dada, explícitamente estaría realizando una tarea de fluidez verbal (FV). Aunque la FV es una tarea de producción que requiere la puesta en marcha de los procesos subyacentes de acceso al léxico, implica también la habilidad de organización cognitiva, la capacidad de llevar a cabo una búsqueda no habitual de palabras, atención focal, atención sostenida y procesos de inhibición, entre otros. Todos estos mecanismos forman parte de un conjunto que se ha denominado funciones ejecutivas. Las funciones ejecutivas son las actividades cognitivas encargadas de la anticipación y el establecimiento de metas, la formación de planes y programas, el inicio de las actividades y operaciones mentales, de la organización temporal y secuenciación, comparación, clasificación y categorización, la autorregulación de las tareas, y la habilidad de llevarlas a cabo eficientemente. Por lo tanto, definen la actividad de un conjunto de procesos cognitivos vinculada al funcionamiento coordinado de los sistemas corticales y subcorticales de los lóbulos frontales (Ettlin y Kischka, 1998; Stuss, 1992).

La evaluación de la FV se remonta a las primeras décadas del siglo pasado, así por ejemplo, encontramos el trabajo de Thurstone (1938) donde se evaluaba la FV de forma oral y escrita, y desde que Borkwski, Benton y Spreen (1967) propusieran la necesidad de evaluar la FV en personas con daño cerebral, se considera que las medidas de FV deben ser incluidas en cualquier protocolo de evaluación neuropsicológica (Heaton, Miller, Tayllor y Grant, 2004; Huff, 1990). Una de las principales razones es que la FV constituye un buen indicador del funcionamiento ejecutivo (Henry y Crawdford, 2004) en la medida que viene definida como, precisión en la búsqueda, uso de estrategias, actualización de la información y la producción de elementos en un tiempo dado, aspectos vinculados al córtex prefrontal (Lezak, Howieson y Loring, 2004).

La FV es medida habitualmente a través de pruebas donde se solicita al sujeto que produzca la mayor cantidad de palabras pertenecientes a una categoría restringida dentro de un tiempo limitado (generalmente 60 segundos). Por norma general, la evaluación de la FV se divide en dos pruebas: (1) Una denominada fluidez verbal semántica (FVS), donde se pide al sujeto que nombre todos los elementos dentro de una categoría semántica determinada (p.ej. animales, frutas); (2) y otra prueba denominada fluidez verbal fonológica (FVF), en la que se pide al sujeto que diga todas las palabras que comiencen con una letra particular (p.ej. F, A, S). Si bien ambas pruebas exigen una serie de demandas ejecutivas, se ha sugerido que no implican los mismos procesos y estrategias cognitivas. En la tarea FVS la evocación de palabras se fundamenta principalmente en llevar a cabo asociaciones semánticas y en el significado de las palabras. Dependerían de la memoria y del conocimiento semántico (Henry y Crawdford, 2004). La FVF requiere el uso de estrategias no habituales (Perret, 1974) ya que buscar palabras por letra inicial no es una tarea común e implica un mayor esfuerzo 
(Hurks et al., 2006). Además, la FVF exige la inhibición de la respuesta incorrecta lo que representa un mayor esfuerzo cognitivo.

Diversos estudios han delimitado los correlatos neuroanatómicos asociados a la FVF y a la FVS. Martín et al. (1994) haciendo uso del paradigma de tareas concurrentes, concluyen que la FVF está asociada a córtex frontal y la FVS asociada al córtex temporal. De forma similar Gourovitch et al. (2000), a través de técnicas de neuroimagen encuentran una mayor actividad en el lóbulo frontal anterior en las tareas de FVF y una mayor activación del córtex temporal en la tarea de FVS. Sin embargo, estudios llevados con pacientes con lesiones en el lóbulo frontal (Baldo y Shimamura, 1998; Schwartz y Baldo, 2001) o el reciente meta-análisis llevado a cabo por Henry y Crawdford (2004) encuentran que en los pacientes con daño en el lóbulo frontal las alteraciones en ambas tareas son comparables.

La necesidad de llevar a cabo la evaluación de las funciones ejecutivas en los sujetos con Déficit de Atención/Hiperactividad (TDAH), surge a partir de los estudios que involucran a los lóbulos frontales en dicho trastorno (por ejemplo, Barkley, 1997a, 1997b). Numerosas investigaciones concluyen que existe una disfunción en el sistema regulador fronto-estriatal en los sujetos con TDAH (Heilman, Voeller y Nadeau, 1991; Lou, Heriksen y Bruhn, 1989; Zatmekin y Rapoport, 1986). Finalmente, el advenimiento de las nuevas técnicas de neuroimagen, ha permitido constatar en el TDAH la implicación de la corteza frontal tanto a nivel estructural (Casey et al., 1997; Castellanos et al., 1996; Filipek et al., 1996) como funcional (Casey et al., 1997; Castellanos, 1997; Rubia et al., 1999). Estos hallazgos permiten postular la hipótesis de que los trastornos de las habilidades verbales en los niños con TDAH están relacionados con alteraciones subyacentes de actividades cognoscitivas de alto nivel. Sin embargo, los estudios que evalúan la FV en el TDHA no son del todo concluyentes. Mientras que en las tareas de FVS los niños con TDHA no se distinguen de los controles (Sergeant, Geurts y Oostelaan, 2002), en las tareas FVF existen resultados divergentes, algunos estudios informan de una pobre ejecución en los sujetos con TDAH (Grodzinsky y Diamond, 1992; Koziol y Stout, 1992; Pineda, Ardila y Rosselli, 1999), otros no encuentran diferencias (Fischer, Barkley, Edelbrock y Smallish, 1990; Schuerholz, Singer y Denckla, 1998; Shallice et al., 2002).

Una cuestión que debemos considerar en el uso de medidas de fluidez verbal con poblaciones más jóvenes es la relación con el desarrollo evolutivo. Existen evidencias que ponen de manifiesto que durante la infancia y adolescencia las puntuaciones en las pruebas de fluidez verbal aumentan con la edad (Brocki y Bohlin, 2004; Matute, Rosselli, Ardila y Morales, 2004) y, debido a la mayor dificultad de la tarea fonológica, se observa un menor incremento de ésta al compararse con la tarea semántica (Koren, Kofman y Berger, 2004). Aunque las medidas fluidez verbal parecen ser sensibles al desarrollo neurológico, aún no está claro a qué edad el rendimiento en 
estos instrumentos alcanza los niveles adultos. Algunos autores han encontrado que los niños de 10 años ya logran un nivel equivalente al del adulto en pruebas de fluidez (Anderson, Northam, Hendy y Wrenall, 2001). Sin embargo, para otros la fluidez verbal parece alcanzar su máximo desarrollo entre la adolescencia y la adultez temprana (Klenberg, Korkman y Lahti-Nuuttila, 2001). A su vez, Matute et al. (2004) y Hurks et al. (2006) afirman que las habilidades semánticas y fonológicas no alcanzan el nivel adulto de forma conjunta, mientras que en las habilidades semánticas se alcanzarían el nivel del adulto entre los 14 y los 15 años, en las habilidades de fluidez fonológica se alcanzarían posteriormente. Debemos tener en cuenta la existencia de variables como el nivel educativo y el medio sociocultural que pueden influir tanto en el desempeño de diversas pruebas neuropsicológicas como en la modificación de la organización funcional cerebral que ocurre cuando el sujeto se expone al aprendizaje de la lectoescritura. Se ha demostrado que los sujetos con menor nivel de vocabulario obtienen peores puntuaciones en pruebas de fluidez verbal (Ruff, Light, Parker y Levin, 1997), que el nivel educativo ejerce una mayor influencia sobre la fluidez fonológica que la semántica (Ratcliff et al., 1998) o que, un bajo nivel educativo de los padres ha sido asociado con una baja producción de palabras en el niño en pruebas de fluidez verbal (Ardila, Rosselli, Matute y Guajardo, 2005).

Debido a que las funciones frontales están aún en desarrollo durante la etapa infantil, y que las tareas de FV aportan valiosa información acerca de los procesos y estrategias de búsqueda léxica, capacidad de almacenamiento en la memoria semántica, y en las estrategias vinculadas al funcionamiento ejecutivo, nuestro principal objetivo era estudiar la ejecución en función de la edad en las dos variantes de la tarea. Analizaremos el patrón evolutivo de la fluidez verbal en la población infantil de habla española así como poner a disposición de los profesionales de datos normativos de considerable interés clínico. Estos datos permitirán valorar la fluidez verbal con tareas que han demostrado sensibilidad para evaluar el funcionamiento ejecutivo. Hicimos uso de las tareas que habitualmente han sido utilizadas para evaluar las FV. Empleamos la categoría animales para la evaluación de la FVS, y las consignas F, A y M para evaluar la FVF. La sustitución de la letra $\mathrm{S}$ (que es la más habitual en los estudios) por la letra $\mathrm{M}$ vino justificada por el fenómeno de seseo presente en diversas regiones hispanohablantes, donde los fonemas $[\theta]$ y [s] no se diferencian en la producción oral. Tal y como afirman Nieto, Galtier, Barroso y Espinosa (2008) este inconveniente es especialmente relevante en el caso del estudio de la FVF en niños, dado que los procesos implicados en la búsqueda y selección léxica pueden estar afectados por el habla y el nivel de consolidación del conocimiento ortográfico del sujeto. 


\section{MÉTODO}

\section{Participantes}

Se seleccionó una muestra de 1.032 sujetos (510 niños y 522 niñas) de edades comprendidas entre los 6 años y 3 meses y los 12 años y 4 meses, cuya lengua materna era el español, no presentaban daño neurológico, psiquiátrico o dificultades de aprendizaje y no estaban en el estadillo de educación especial. La muestra total fue dividida en 6 grupos en función de la edad: (1) de 6 años y 3 meses a 7 años y 4 meses; (2) de 7 años y 5 meses a 8 años y 4 meses; (3) de 8 años y 5 meses a 9 años y 4 meses; (4) de 9 años y 5 meses a 10 años y 4 meses; (5) de 10 años y 5 meses a 11 años y 4 meses; (6) de 11 años y 5 meses a 12 años y 4 meses. La distribución de la muestra en función del género se presenta en la tabla 1.

Tabla 1. Distribución de la muestra de estudio según edad y sexo

\begin{tabular}{cccc}
\hline Grupos & Edad (años,meses) & Niño & Niña \\
\hline Grupo 1 & $6.3-7.4$ & 101 & 103 \\
\hline Grupo 2 & $7.5-8.4$ & 87 & 80 \\
\hline Grupo 3 & $8.5-9.4$ & 90 & 89 \\
\hline Grupo 4 & $9.5-10.4$ & 84 & 72 \\
\hline Grupo 5 & $10.5-11.4$ & 66 & 98 \\
\hline Grupo 6 & $11.5-12.4$ & 82 & 80 \\
\hline TOTAL & & 510 & 522 \\
\hline
\end{tabular}

\section{Instrumentos}

Tarea de Fluidez Verbal Semántica (FVS): La tarea solicitaba a los participantes que dijeran el mayor número posible de "animales" durante un minuto.

Tarea de Fluidez Verbal Fonológica (FVF): se midió pidiendo a los participantes que dijeran el mayor número posible de palabras durante un minuto que comenzasen por una letra determinada. Las letras empleadas fueron F, A y M. La media de las tres consignas dio lugar a la variable FVF. En ambas tareas y durante las instrucciones se advertía al participante que los nombres propios, las siglas, marcas comerciales y las palabras derivadas o diferentes formas de un mismo verbo, no se considerarían como respuestas válidas.

\section{Diseño y procedimiento}

Utilizamos un diseño transversal donde la variable independiente fueron los grupos establecidos en función del rango de edad y como variables dependientes el rendimiento en las pruebas de FVS y FVF. La evaluación fue llevada a cabo por Licenciados en Psicología que fueron previamente entrenados. La administración de las pruebas fue individual y se llevaron a cabo en un aula suficientemente iluminada y carente de ruidos del Centro. 


\section{RESULTADOS}

Antes de llevar a cabo los distintos estudios, comprobamos que la distribución de la muestra en función de la variable sexo en cada grupo de edad era similar $\left[\chi_{(5)}^{2}=7.37 ; p=0.194\right]$.

\section{A. Estudio de baremación}

El objetivo de este estudio es disponer de una escala normativa que permita determinar la posición que ocupa la puntuación directa que obtenga un alumno de infantil en relación con los que obtienen las personas que integran nuestro grupo normativo. Con este fin, se calcularon los percentiles en cada grupo de edad a partir de los datos obtenidos por la muestra de estudio, siendo FVF la suma de las puntuaciones directas de las consignas F, A y M. Las puntuaciones para los percentiles 75, 50 y 25 para cada grupo de edad tanto en FVS (animales), FVF como para cada una de las consignas fonológicas pueden consultarse en la tabla 2.

Tabla 2. Baremos para las consignas F, A, M, Fluidez Verbal Fonológica y Fluidez Verbal Semántica

\begin{tabular}{|c|c|c|c|c|c|c|}
\hline \multirow{2}{*}{$\begin{array}{c}\text { Grupos } \\
\text { (años;meses) }\end{array}$} & \multicolumn{6}{|c|}{ Tareas } \\
\hline & Percentil & $\mathrm{F}$ & $\mathrm{A}$ & $\mathrm{M}$ & FVF & FVS \\
\hline \multirow{3}{*}{$\begin{array}{l}\text { Grupo } 1 \\
(6 ; 3-7 ; 4)\end{array}$} & 25 & 2 & 2 & 2 & 7 & 8 \\
\hline & 50 & 3 & 4 & 4 & 10 & 10 \\
\hline & 75 & 4 & 5 & 6 & 14 & 13 \\
\hline \multirow{3}{*}{$\begin{array}{l}\text { Grupo } 2 \\
(7 ; 5-8 ; 4)\end{array}$} & 25 & 3 & 3 & 3 & 9 & 10 \\
\hline & 50 & 4 & 5 & 5 & 15 & 13 \\
\hline & 75 & 6 & 7 & 7 & 20 & 16 \\
\hline \multirow{3}{*}{$\begin{array}{l}\text { Grupo } 3 \\
(8 ; 5-9 ; 4)\end{array}$} & 25 & 4 & 4 & 5 & 13 & 12 \\
\hline & 50 & 5 & 5 & 6 & 17 & 14 \\
\hline & 75 & 6 & 7 & 8 & 20 & 17 \\
\hline \multirow{3}{*}{$\begin{array}{c}\text { Grupo } 4 \\
(9 ; 5-10 ; 4)\end{array}$} & 25 & 4 & 4 & 5 & 14 & 13 \\
\hline & 50 & 6 & 6 & 7 & 19 & 16 \\
\hline & 75 & 7 & 8 & 9 & 24 & 19 \\
\hline \multirow{3}{*}{$\begin{array}{c}\text { Grupo } 5 \\
(10 ; 5-11 ; 4)\end{array}$} & 25 & 4 & 6 & 6 & 17 & 13 \\
\hline & 50 & 6 & 7 & 8 & 21 & 16 \\
\hline & 75 & 8 & 9 & 10 & 26 & 19 \\
\hline \multirow{3}{*}{$\begin{array}{c}\text { Grupo } 6 \\
(11 ; 5-12,4)\end{array}$} & 25 & 6 & 6 & 7 & 19 & 15 \\
\hline & 50 & 7 & 8 & 9 & 23 & 17 \\
\hline & 75 & 9 & 10 & 11 & 30 & 20 \\
\hline
\end{tabular}

\section{B. Perfil evolutivo (análisis de tendencia)}

Se llevaron a cabos sendos ANOVA para cada una de las variables dependientes del estudio (FVS y FVF) tomando como factor con 6 niveles a los grupos establecidos en función de los rangos de edad. A su vez se realizó un análisis de tendencia, y debido a la disparidad del tamaño de los grupos, se consultó la solución ponderada. 
En la tabla 3 se recoge las medias y desviaciones típicas del número de palabras evocadas por los participantes ante una consigna dada en el período de un minuto.

Tabla 3. Medias y desviaciones típicas para cada rango de edad en la evocación de palabras en las tareas de Fluidez verbal

\begin{tabular}{|c|c|c|c|c|c|c|c|c|c|c|}
\hline \multirow{3}{*}{$\begin{array}{c}\text { Grupos } \\
\text { (años; meses) }\end{array}$} & \multicolumn{10}{|c|}{ Tareas } \\
\hline & \multicolumn{2}{|c|}{$\mathrm{F}$} & \multicolumn{2}{|c|}{$\mathrm{A}$} & \multicolumn{2}{|c|}{$\mathrm{M}$} & \multicolumn{2}{|c|}{ FVF } & \multicolumn{2}{|c|}{ FVS } \\
\hline & $M$ & $D T$ & $M$ & $D T$ & $M$ & $D T$ & $M$ & $D T$ & $M$ & $D T$ \\
\hline Grupo $1(6 ; 3-7 ; 4)$ & 3.32 & 2.17 & 3.81 & 2.20 & 4.25 & 2.54 & 11.29 & 5.73 & 10.82 & 6.59 \\
\hline Grupo $2(7 ; 5-8 ; 4)$ & 4.57 & 2.38 & 5.04 & 2.75 & 5.43 & 2.59 & 14.95 & 6.578 & 13.11 & 3.92 \\
\hline Grupo $3(8 ; 5-9 ; 4)$ & 5.14 & 2.16 & 5.48 & 2.31 & 6.43 & 2.57 & 16.99 & 5.83 & 14.15 & 3.81 \\
\hline Grupo $4(9 ; 5-10 ; 4)$ & 5.61 & 2.44 & 6.21 & 2.75 & 7.18 & 2.81 & 19.01 & 6.77 & 15.67 & 4.01 \\
\hline Grupo $5(10 ; 5-11 ; 4)$ & 6.37 & 2.54 & 7.29 & 2.75 & 7.96 & 2.89 & 21.60 & 6.78 & 16.20 & 4.12 \\
\hline Grupo $6(11 ; 5-12,4)$ & 7.45 & 2.86 & 8.01 & 3.01 & 8.72 & 2.85 & 24.20 & 7.18 & 17.79 & 4.20 \\
\hline TOTAL & 5.32 & 2.77 & 5.88 & 2.98 & 6.56 & 3.10 & 17.70 & 7.76 & 14.45 & 5.17 \\
\hline
\end{tabular}

Tabla 4. Resultados del análisis de los contrastes a posteriori de las diferencias par a par para la variable rango-edad en Fluidez Verbal Fonológica y Fluidez Verbal Semántica (Corrección de Bonferroni)

\begin{tabular}{|c|c|c|c|c|c|c|c|c|c|c|c|c|c|c|c|c|}
\hline & & \multicolumn{15}{|c|}{ Grupos } \\
\hline & & \multicolumn{3}{|c|}{ Grupo 2} & \multicolumn{3}{|c|}{ Grupo 3} & \multicolumn{3}{|c|}{ Grupo 4} & \multicolumn{3}{|c|}{ Grupo 5} & \multicolumn{3}{|c|}{ Grupo 6} \\
\hline & & $t$ & $g l$ & $p$ & $t$ & $g l$ & $p$ & $t$ & $g l$ & $p$ & $t$ & $g l$ & $p$ & $t$ & $g l$ & $p$ \\
\hline \multirow{4}{*}{ FVF } & Grupo 1 & 5.42 & 318 & $<.001 *$ & 8.6 & 327 & $<.001 *$ & 11.22 & 303 & $<.001^{*}$ & 15.21 & 315 & $<.001^{*}$ & 18.99 & 295 & $<.001 *$ \\
\hline & Grupo 3 & & & & & & & 2.85 & 284 & $=.067$ & 6.60 & 296 & $<.001^{*}$ & 10.3 & 276 & $<.001^{*}$ \\
\hline & Grupo 4 & & & & & & & & & & 3.59 & 272 & $<.01^{*}$ & 7.17 & 252 & $<.001 *$ \\
\hline & Grupo 5 & & & & & & & & & & & & & 3.64 & 264 & $<.001^{*}$ \\
\hline \multirow{4}{*}{ FVS } & Grupo 2 & & & & 2.09 & 299 & $=.551$ & 4.97 & 275 & $<.001^{*}$ & 6.07 & 287 & $<.001^{*}$ & 9.16 & 267 & $<.001^{*}$ \\
\hline & Grupo 3 & & & & & & & 2.99 & 284 & $=.042 *$ & 4.09 & 296 & $<.001^{*}$ & 7.24 & 276 & $<.001^{*}$ \\
\hline & Grupo 4 & & & & & & & & & & 1.18 & 272 & $=1.00$ & 4.07 & 252 & $<.001^{*}$ \\
\hline & Grupo 5 & & & & & & & & & & & & & 3.09 & 264 & $=.030^{*}$ \\
\hline
\end{tabular}

Nota: Grupo 1=6;3-7;4; Grupo 2= 7;5-8;4; Grupo 3= 8;5-9;4; Grupo 4= 9;5-10;4; Grupo5=10;5-11;4 Grupo 6=11;5-12;4 (años;meses)

Figura 1. Perfil de Desarrollo de la Frecuencia Verbal Semántica y de la Frecuencia Verbal Fonológica

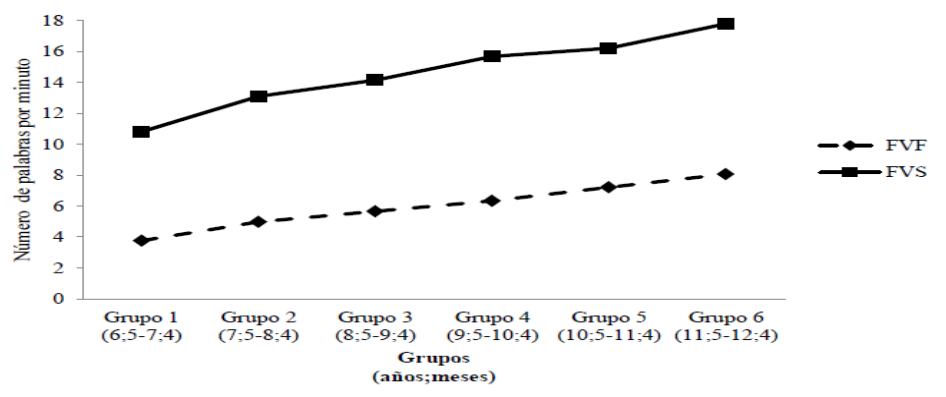

El análisis de varianza para la FVS muestra que existen diferencias significativas en función de los distintos grupos de edad $\left[F_{(5,1026)}=51.65 ; p<.001\right.$, 
$\left.\omega^{2}=.246\right]$. Esta diferencia también fue encontrada en FVF $\left[F_{\text {asintótica }(5,469.803)}=93.50 ; p<\right.$ $\left..001 ; \omega_{\text {estimada }}^{2}=.246\right]$. La tabla 4 recoge las diferencias significativas de los análisis a posteriori de las diferencias par a par entre los diferentes grupos de edad y el número de palabras evocadas por minuto en cada una de las tareas. Los análisis de tendencia demostraron la existencia una relación lineal ascendente entre las variables de agrupamiento para la FVS $\left[F_{(1,1026)}=251.40 ; p<.001\right]$ y para $\operatorname{FVF}\left[F_{(5,110,25)}=459.83\right.$; $p<.001]$. En la figura 1 se muestra la representación gráfica de estos resultados.

\section{DISCUSIÓN}

El objetivo principal de este estudio ha sido proporcionar datos normativos para la población infantil de habla española en las variantes más utilizadas para evaluar la FV. Las pruebas de FV son instrumentos que cuentan con un gran número de estudios descritos en la bibliografía y su fácil uso los convierte en instrumentos atractivos para la clínica y para la investigación en Neuropsicología en diferentes grupos de edad. Los datos obtenidos permitirán detectar una alteración en la fluidez bajo una directriz fonológica o léxica.

En cuanto al estudio del desarrollo evolutivo de FVS y de FVF observamos un incremento lineal en el rendimiento mostrado por los escolares en función de la edad. Estos resultados son coincidentes con los estudios de Brocki y Bohlin, (2004) y Matute et al. (2004), quienes afirman que durante la etapa infantil a medida que aumenta la edad aumenta la ejecución las tareas de fluidez. Los análisis posteriores nos llevan a entender posibles patrones de desarrollo de esta habilidad. En este sentido, los contrastes par a par (véase tabla 3) reflejaron que en ambas tareas, el grupo de mayor edad se diferenció significativamente del resto de los grupos. Por lo tanto, podemos concluir que durante el período de edades comprendido entre 6 a 12 años tanto la FVS y FVF están en período de constante desarrollo, aunque no podemos determinar en qué momento se alcanza el nivel de ejecución similar al adulto. Aunque en general se observa un mayor rendimiento en función de la edad, en la FVF se observa que no existían diferencias estadísticamente significativas entre el grupo 3 y el grupo 4 , y debido a que si se diferenciaban del grupo 5 podemos decir que ambos grupos se comportan de forma similar, y no se pone de manifiesto una mejoría en ese período de edad. En la FVS encontramos que primero el grupo dos y el grupo 3 los que no se diferencian entre sí (pero ambos si se diferencian del grupo 4), y el grupo 4 y el grupo 5 tampoco se diferencian entre sí pero si con el grupo 6. Una explicación a estos períodos de meseta es que los niños están en la etapa de desarrollo de algunas funciones ejecutivas, por lo que con se pueden observar rendimientos similares en algunas funciones en niños entre 8 y 10 años (Pineda, 2000).

La importancia del estudio radica en disponer de datos normativos y del perfil de desarrollo de la prueba de FV en la etapa infantil, que es el período de mayor 
desarrollo de las funciones ejecutivas. Los niños de 12 años ya tienen una organización cognoscitiva cercana a los adultos; aunque el desarrollo completo de dichas funciones se alcanza alrededor de los 16 años (Chelune, Fergunson, Koon y Dickey, 1986; Levin et al., 1991; Welsh, Pennington y Groisser, 1991). Estos resultados proporcionan información útil para la práctica clínica. Así por ejemplo, es sabido que uno de los trastornos más frecuentes en la infancia es el trastorno por déficit de atención con hiperactividad. Los niños con TDAH, además de las dificultades en el manejo de sus recursos atencionales que los caracterizan, manifiestan problemas en las funciones ejecutivas (Barkley, 2003; Rubia et al., 2001). De acuerdo con lo señalado por Pineda et al. (1999), las pruebas de FV ponen de manifiesto eficientemente las dificultades cognitivas de los individuos que presentan TDAH ya que un bajo rendimiento podrían ser expresiones de una disfunción ejecutiva, subyacente a una deficiencia en la maduración de los sistemas prefrontales.

\section{Agradecimientos}

Esta investigación ha sido financiada por la Agencia Canaria de Investigación, Innovación y Sociedad de la Información de la Consejería de Educación, Universidades y Sostenibilidad del Gobierno de Canarias, con ref. PI 2007/012 del que es IP el cuarto autor.

\section{REFERENCIAS}

Anderson, V., Northam, E., Hendy, J. y Wrenall, J. (2001). Developmental Neuropsychology: A clinical approach. New York: Psychology Press.

Ardila, A., Rosselli, M., Matute, E. y Guajardo, G. (2005). The influence of parents educational level on the development of executive functions. Developmental Neuropsychology, 28, 539-560.

Baldo, J.V. y Shimamura, A.P. (1998). Letter and category fluency in patients with frontal lobe lesions. Neuropsychology, 12, 259-267.

Barkley, R.A. (1997a). Behavioral inhibition sustained, attention, and executive functions: Constructing a unifying theory of ADHD. Psychological Bulletin, 121, 65-94.

Barkley, R.A. (1997b). ADHD and the nature of self-control. New York: Guilford.

Barkley, R.A. (2003). Issues in the diagnosis of attention-deficit/hyperactivity disorder in children. Brain Development, 25, 77-83.

Borkowski, J.G., Benton, A.L. y Spreen, O. (1967). Word fluency and brain damage Neuropsychologia, 5, 135-140.

Brocki, K.C. y Bohlin, G. (2004). Executive functions in children aged 6 to 13: A dimensional and developmental study. Developmental Neuropsychology, 26, 571-593.

Casey, B.J., Castellanos, F.X., Giedd, J.N., Marsh, W.L., Hamburger, S.D., Schubert, A.B., Vauss, Y.C., Vaituzis, A.C., Dickstein, D.P., Sarfatti, S.E. y Rapoport, J.L. (1997). Implication of right frontstriatal circuitry in response inhibition and attention-deficit/hyperactivity 
disorder. Journal of the American Acdemy of Child and Adolescent Psychiatry, 36, 374383.

Castellanos, F.X., Giedd, J.N., Marsh, W.L., Hamburger, S.D., Vaituzis, A.C., Dickstein, D.P., Sarfatti, S.E., Vauss, Y.C., Snell, J.W., Lange, N., Kaysen, D., Krain, A.L., Ritchhie, G.F., Rajapakse, J.C. y Rapoport, J.L. (1996). Quantitative brain magnetic resonance imaging in attention-deficit hyperactivity disorder. Archives of General Psychiatry, 53, 607-616.

Chelune, G.J., Ferguson, W., Koon, R. y Dickey, T.O. (1986). Frontal lobe desinhibition in attention deficit disorder. Child Psychiatry and Human Development, 16, 221-234.

Ettlin T. y Kischka, U. (1998). Beside frontal lobe testing. The frontal lobe score. En B.L. Miller y J.L. Cummings (eds.), The human frontal lobes (pp. 233-246). Nueva York: The Guilford Press.

Filipek, P.A., Semrud-Clikeman, M., Steingard, R.J., Renshaw, P.F., Kennedy, D.N. y Biederman, J. (1997). Volumetric Mri analysis comparing subjects having attention-deficit hyperactivity disorder with normal controls. Neurology, 48, 589-601.

Fischer, M., Barkley, R.A., Edelbrock, C.S. y Smallish, L. (1990). The adolescent outcome of hyperactive children diagnosed by research criteria, II: Academic, attentional, and neuropsychological status. Journal of Consulting and Clinical Psychology, 58, 580-588.

Gourovitch, M.L., Kirkby, B.S., Goldberg, T.E., Weinberger, D.R., Gold, J.M., Esposito, G., Van Horn, J.D. y Berman, K.F. (2000). A comparison of rCBF patterns during letter and semantic fluency. Neuropsychology. 14, 353-360.

Grodzinsky, G.M. y Diamond, R. (1992). Frontal lobe functioning in boys with attention-deficit hyperactivity disorder. Developmental Neuropsychology, 8, 427-445.

Heaton, R.K., Miller, S.W., Taylor, M.J. y Grant, I. (2004). Revised comprehensive norms for an expanded Halstead-Reitan battery (norms, manual and computer program). Odessa, FL: Psychological Assessment Resources.

Heilman, K.M., Voeller, K.K.S. y Nadeau, S.E. (1991). A possible pathophysiological substrate of attention deficit hyperactivity disorder. Journal of Child Neurology, 6, 74-79.

Henry, J.D. y Crawford, J.R. (2004). A meta-analytic review of verbal fluency performance in traumatic brain injured patients. Neuropsychology, 18, 621-628.

Hurks, P.P.M., Vles, J.S.H., Hendriksen, J.G.M., Kalff, A.C., Feron, F.J.M., Kroes, M., et al. (2006). Semantic category fluency versus initial letter fluency over 60 seconds as a measure of automatic and controlled processing in healthy school-aged children. Journal of Clinical and Experimental Neuropsychology, 28, 684-695.

Huff, F.J. (1990). Language in normal aging and age-related neurological diseases. En F. Boller y J. Grafman (eds.), Handbook of Neuropsychology. Vol. 4. Elsevier Science Publishers B.V.

Klenberg, L., Korkman, M. y Lahti-Nuuttila, P. (2001). Differential development of attention and executive functions in 3 to 12 years-old Finnish children. Developmental Neuropsychology, 20, 407-428.

Koren, R., Kofman, O. y Berger, A. (2005). Analysis of word clustering in verbal fluency of school-aged children. Archives of Clinical Neuropsychology, 20, 1087-1104.

Koziol, L.F. y Stout, C.E. (1992). Use of a verbal fluency measure in understanding and evaluating ADHD as an executive function disorder. Perceptual and Motor Skills, 75, 1187-1192. 
Levin, H.S., Culhane, K.A., Hartman, J., Evankovich, K., Mattson, A.J., Hardward, H., Ringholz, G., Ewing-Cobbs, L. y Fletcher, J.M. (1991). Developmental changes in performance on tests of pruported frontal lobe functioning. Developmental Neuropsychology, 7, 377-395.

Lezak, M.D., Howieson, D.B. y Loring, D.W. (2004). Neuropsychological assessment. New York: Oxford University Press.

Lou, H.C., Henriksen, L., Bruhn, P., Borner, H. y Nielsen, J.B. (1989). Striatal dysfunction in attention deficit and hyperkinetic disorder. Archives of Neurology, 46, 48-52.

Matute, E., Rosselli, M., Ardila, A. y Morales, L. (2004). Verbal and non-verbal fluency in Spanish speaking children. Developmental Neuropsychology, 26, 647-660.

Nieto, A., Galtier, I., Barroso, J. y Espinosa, G. (2008). Fluencia verbal en niños españoles en edad escolar: estudio normativo piloto y análisis de las estrategias organizativas. Revista de Neurología, 46, 2-6.

Perret, E. (1974). The left frontal lobe of man and the suppression of habitual responses in verbal categorical behaviour. Neuropsychologia, 12, 323-330.

Pineda, D. (2000) La función ejecutiva y sus trastornos. Revista de Neurología, 30, 764-768.

Pineda, D., Ardila, A. y Rosselli, M. (1999). Neuropsychological and behavioral assessment of ADHD in seven to twelve years-old children: A discriminant analysis. Journal of Learning Disabilities, 32, 159-173.

Ratcliff, G., Ganguli, M., Chandra, V., Sharma, S., Belle, S., Seaberg, E., et al. (1998). Effects of literacy and education on measures of word fluency. Brain and Language. 61, 115-22.

Rubia, K., Taylor, E., Smith, A.B., Oksannen, H., Overmeyer, S. y Newman, S. (2001). Neuropsychological analyses of impulsiveness in childhood hyperactivity. The British Journal of Psychiatry, 179, 138-43.

Rubia, K., Overmeyer, S., Taylor, E., Brammer, M., Williams, S.C.R., Simmons, A. y Bullmore, E.T. (1999). Hypofrontality in attention deficit hyperactivity disorder during higher-order motor control: A study with functional MRI. American Journal of Psychiatry, 156, 891896.

Ruff, R.M., Light, R.H., Parker, S.B. y Levin, H.S. (1997). The psychological construct of word fluency. Brain and Language, 57, 394-405.

Schuerholz, L.J., Singer, H.S. y Denckla, M.B. (1998). Gender study of neuropsychological and neuromotor function in children with Tourette syndrome with and without attention-deficit hyperactivity disorder. Journal of Child Neurology, 13, 277-282.

Schwartz, S. y Baldo, J. (2001). Distinct patterns of word retrieval in right and left frontal lobe patients: a multidimensional perspective. Neuropsychologia. 39, 1209-1217.

Sergeant, J.A., Geurts, H. y Oosterlaan, J. (2002). How specific is a deficit of executive functioning for attention-deficit hyperactivity disorder? Behavioural Brain Research, 130, 3-28.

Shallice, T., Marzocchi, G.M., Coser, S., Del Savio, M., Meuter, R.F. y Rumiati, R.I. (2002). Executive function profile of children with Attention Deficit Hyperactivity Disorder. Developmental Neuropsychology, 21, 43-71.

Stuss, D. (1992). Biological and psychological development of executive functions. Brain and Cognition, 20, 8-23.

Thurstone, L.L. (1938). Primary Mental Abilities. Chicago: University of Chicago Press. 
Welsh, M.C., Pennington, B.F. y Groiser, D.B. (1991). A normative develpomental study of executive function: A window on prefrontal function in children. Developmental Neuropsychology, 7, 131-149.

Zametkin, A.J. y Rapoport, J.L. (1986). The pathophysiology of attention deficit disorder with hyperactivity: A review. En B.B. Lahey y A.E. Kazdin (Eds.), Advances in clinical child psychology (pp. 177-216). Nueva York: Plenum.

Recibido: 30 de septiembre de 2011

Recepción Modificaciones: 20 de marzo de 2012

Aceptado: 22 de marzo de 2012 